\title{
Evaluación epidemiológica de plaguicidas inhibidores de acetilcolinesterasa en Colombia, 1996-1997
}

\author{
Elizabeth Silva, Ligia Morales, Jaime E. Ortiz \\ Laboratorio de Salud Ambiental, Instituto Nacional de Salud, Bogotá, D.C., Colombia
}

\begin{abstract}
El programa de vigilancia epidemiológica de plaguicidas organofosforados y carbamatos (VEO) fue creado mediante el establecimiento de convenios de cooperación y asistencia técnicocientífica entre el Laboratorio de Salud Ambiental del Instituto Nacional de Salud y las seccionales de salud del país. El programa tiene como finalidad detectar precozmente los casos de absorción elevada de plaguicidas e impulsar el desarrollo de acciones de promoción, prevención y control, que disminuyan la incidencia de intoxicaciones agudas y los efectos crónicos producidos por los plaguicidas inhibidores de la acetilcolinesterasa (ACHE). Durante 1996 y 1997, participaron en el estudio 17 entidades territoriales de salud y 21.454 personas que presentaban riesgo de exposición a plaguicidas organofosforados y carbamatos. A estas personas se les realizaron 24.167 análisis de ACHE, mediante el método de Limperos y Ranta, utilizando el equipo de Lovibond. La prevalencia de valores anormales de ACHE fue de $6,1 \%$, muy similar a las encontradas de 1993 a 1995. Las entidades territoriales de salud encontradas con mayores prevalencias después de realizar la estandarización directa fueron Bolívar $(20,3 \%)$ en 1996 y Córdoba $(17,7 \%)$ en 1997. Las variables asociadas con mayores prevalencias de anormalidad para ACHE fueron las aplicaciones aérea y terrestre en actividades económicas; el de banderero y mezclador-tanqueador-formulador en oficios y los grupos de edad de 18 a 25 años y de 6 a11 años.
\end{abstract}

Solamente $36,1 \%$ de los participantes en el estudio estaban afiliados al sistema general de seguridad social en salud (SGSSS); $63,9 \%$ no estaban cubiertos por el sistema y, de éstos, 7,1\% tenía valores anormales de ACHE. La evaluación epidemiológica para 1996-1997 se comparó con la de los años anteriores y se determinó la población con mayor riesgo; se cuantificaron los niveles de intoxicación y se aportaron las conclusiones para fortalecer la vigilancia epidemiológica. De esta manera, a través de sus actividades y de su trayectoria, el programa VEO ha ido perfilando con mayor exactitud el riesgo ocasionado por los plaguicidas inhibidores de la ACHE en el país.

Palabras clave: vigilancia epidemiológica, plaguicidas, organofosforados, carbamatos, acetilcolinesterasa, intoxicación, contaminación ambiental.

\section{Epidemiological evaluation of acetylcholinesterase-inhibiting pesticides in Colombia, 1996-1997}

The epidemiological surveillance program for organophosphate and carbamate pesticides (VEO) was created through scientific and technical surveillance and cooperation agreements between the Environmental Health Laboratory at the National Institute of Health and the regional health entities. The programme's aim is the early detection of cases of high pesticide levels and the establishment of priorities for the development of promotion, prevention and control actions, which will decrease the incidence of acute poisoning and the chronic effects produced by acetylcholinesterase-inhibiting pesticides (ACHE).

Seventeen Regional Health Offices ( $\mathrm{RHO}$ ) and 21,454 people with high risk of exposure to organophosphate (OF) and carbamate (C) pesticides participated in this study during 1996 and 1997. The Limperos and Ranta method, using Lovibond equipment, was used for ACHE analysis of 24,167 blood samples. Abnormal ACHE value prevalence was $6.1 \%$, being very similar to that 
found between 1993 and 1995. The RHO with the highest prevalence after direct standardisation were Bolívar (20.3\%) in 1996 and Córdoba (17.7\%) in 1997. The variables associated with greater ACHE abnormality prevalence were: for economical activity, air and terrestrial pesticide application work; for occupation, the banderero (the man who indicates already fumigated areas) and the mixer-sprayer-warehousing; and for ages, the 18 to 25 and 6 to 11 age groups. Only $36.1 \%$ of those participating in the study were affiliated to the social security general health system; $63.9 \%$ were not protected by social security and $7.1 \%$ of these had abnormal ACHE values. Epidemiological evaluation for 1996-1997 was compared with previous years (19931995 ) and the population at highest risk was determined. Poisoning levels were quantified and conclusions were drawn to strengthen epidemiological surveillance. The VEO programme was thus able to define national risk levels caused by ACHE-inhibiting pesticides with much higher accuracy.

Key words: epidemiological surveillance, pesticides, organophosphates, carbamates, acetylcholinesterase, poisoning, environmental pollution.

Aunque el uso de plaguicidas genera beneficios importantes en el desarrollo de la agricultura y, por consiguiente, en la producción de los alimentos, también es la causa de efectos adversos para los humanos, las especies animales y el medio ambiente. Según la OMS, en la primera mitad de los años noventa, hubo 2 a 5 millones de personas intoxicadas por plaguicidas, de las cuales fallecieron 40.000 (1). La situación se agrava más en los países en vías de desarrollo; algunos autores señalan que en estos países, 2 a $3 \%$ de los trabajadores agrícolas sufren algún tipo de intoxicación y fallecen entre 10 y $12 \%$ de ellos (1).

En la actualidad, se utilizan en gran escala los plaguicidas inhibidores de la acetilcolinesterasa (organofosforados y carbamatos), que reemplazan a los organoclorados persistentes. Los organofosforados (OF) son ésteres del ácido fosfórico y ditiofosfórico y su vida media es relativamente corta (horas, días). El grupo de los carbamatos (C) corresponde en su mayor parte a derivados del ácido N-metilcarbámico. Estos dos tipos de plaguicidas pueden penetrar al organismo por inhalación, ingestión, a través de la piel intacta y, también, por las mucosas conjuntival y vaginal. Su biotransformación se realiza mediante enzimas oxidasas, hidrolasas y transferasas y la eliminación se hace principalmente por vía urinaria.

\footnotetext{
Correspondencia:

Elizabeth Silva

esilva@hemagogus.ins.gov.co

Recibido: 14/01/00; aceptado: 19/05/00
}

La acetilcolinesterasa (ACHE) localizada en las terminales nerviosas es el blanco molecular de la toxicidad de los OF y C. Cuando se bloquea la enzima queda impedida para participar en la hidrólisis de la acetilcolina y estos neurotransmisores se acumulan, su acción se intensifica y se producen efectos tóxicos en el sistema nervioso central y en los sistemas parasimpático, simpático y motor (2).

La acción que inhibe la enzima $\mathrm{ACHE}$ es reversible cuando es producida por los $\mathrm{C}$ debido a que su unión con la enzima es lábil. Con los OF, la inhibición de la $\mathrm{ACHE}$ es irreversible debido a que estos compuestos forman una unión covalente, relativamente estable, que impide la regeneración de la enzima libre y activa, por lo cual la restauración de la enzima dependerá de la síntesis de nuevas moléculas de la misma.

Los insecticidas inhibidores de $\mathrm{ACHE}$, junto con los bipiridilos, son la causa de casi todos los casos de intoxicación aguda producidos por el conjunto de los herbicidas, fungicidas e insecticidas (1); específicamente, los OF han sido los causantes de las intoxicaciones masivas con plaguicidas en Colombia (3).

En el mercado existen varios métodos cinéticos para determinar la actividad de la ACHE, los cuales utilizan como matriz el plasma, los eritrocitos o la sangre total. La inhibición de ACHE en plasma es interpretada como un biomarcador de exposición pero no de toxicidad, mientras que la inhibición de la enzima en eritrocitos sí representa un biomarcador de toxicidad (2). El principio de los 
métodos es fundamentalmente el mismo y se basa en el cambio de $\mathrm{pH}$ producido cuando el ácido acético se libera por acción de la ACHE sobre la acetilcolina.

Entre los métodos utilizados, está el electrométrico de Michel en eritrocitos y en plasma (4) que, aunque tiene buena sensibilidad y precisión, requiere un medidor de $\mathrm{pH}$ de escala expandida y procesos como el baño de María. Este equipo, además de aumentar el costo de las pruebas, no es de fácil desplazamiento para la realización del trabajo de campo. En sangre total, están el método electrométrico de Michel y Aldridge modificado (5) y el de Limperos y Ranta (6) modificado por Edson (7); este último es un método colorimétrico visual que se puede utilizar para trabajo de campo con la ayuda de un equipo simple y práctico.

Con el propósito de valorar el método de Limperos y Ranta modificado, el Laboratorio de Salud Ambiental del INS desarrolló un estudio de comparación con el método de Michel y Aldridge modificado. No se encontraron diferencias significativas entre estos dos métodos al ser aplicados a una población expuesta a plaguicidas OF y $\mathrm{C}$ y a una población control del INS.

En el programa de vigilancia epidemiológica VEO se determinan los niveles de ACHE mediante el método de Limperos y Ranta modificado, el cual es muy conveniente por ser un método rápido, de campo y que, además de la rutina, permite atender adecuadamente las emergencias y el trabajo fuera del laboratorio.

En 1993, se realizó en California un programa de evaluación para monitorizar los niveles de ACHE en aplicadores de plaguicidas (8); se utilizó el método de Ellman que también es colorimétrico. Los resultados publicados en este estudio informan $5,4 \%$ de valores de ACHE inferiores al límite permisible, similar al resultado de 4,8\% (26/542) encontrado en un informe anterior del servicio de salud de California en 1989 (8). En ese mismo año, se realizó en Taiwán otro programa de mediciones de ACHE en muestras de plasma de aplicadores de plaguicidas, el cual informó 8,3\% (43/515) de personas con actividad de ACHE inferior al valor límite (8).
A pesar de las fallas de notificación, en los países en vías de desarrollo se informa desde hace varias décadas la gran frecuencia de casos de intoxicación, donde la población ocupacionalmente expuesta es la más afectada. Los estudios de vigilancia epidemiológica en Brasil indican un caso de intoxicación por cada 6 trabajadores agrícolas, con síntomas desde muy leves hasta muy graves (9). En Honduras, en la comunidad vecina a una zona arrocera donde se efectúan rociados aéreos constantes durante todo el año, se encontró que $9,1 \%$ de los pobladores muestreados tenía una disminución de $25 \%$ o más en la actividad de la ACHE (9). En Costa Rica, en un estudio epidemiológico descriptivo, se informó una incidencia anual de $4,5 \%$ intoxicaciones sintomáticas en personas ocupacionalmente expuestas a plaguicidas (10)

En Colombia, aunque las estadísticas sobre intoxicaciones son deficientes, se han registrado tres casos de intoxicaciones masivas causadas por alimentos contaminados con paratión o metilparatión; estas fueron, en 1967, en Chiquinquirá, Boyacá, con más de 500 personas intoxicadas (63 defunciones); en 1970, en Puerto López, Meta, donde hubo 190 intoxicados (7 defunciones), y en 1977 , en Pasto, Nariño, con más de 300 personas intoxicadas (15 defunciones) (3).

Actualmente, y desde 1981, el Laboratorio de Salud Ambiental del Instituto Nacional de Salud está llevando a cabo el Programa de Vigilancia Epidemiológica de Plaguicidas Organofosforados y Carbamatos (VEO), en coordinación con varias entidades territoriales (ETS) del país. En la evaluación del programa, en el cual se analizaron los años de 1993 a 1995, participaron 17 departamentos y se estudiaron 41.899 personas que tenían antecedentes de exposición a plaguicidas OF y C, y se encontró que 6,2\% tenía resultados anormales de ACHE (11). En el marco de este mismo programa, el departamento del Valle del Cauca informó que, durante 1997, habían ocurrido 367 casos de intoxicación aguda por plaguicidas, debidos a exposición ocupacional, voluntaria o accidental (12).

En los Estados Unidos, el mercado mundial de los plaguicidas se valoró en 1994 en U.S. \$25.885 
millones y, en 1996, había aumentado a U.S. $\$ 30.560$ millones. Se pronostica que en los próximos años aumentará en un promedio de 1,6\% hasta alcanzar alrededor de U.S. $\$ 33.000$ millones en el 2001 (1). Según la OMS, aproximadamente $25 \%$ de estas sustancias son utilizadas en los países en vías de desarrollo (1). En nuestro país se ha venido incrementando tanto el consumo como la producción de plaguicidas; en 1994, la producción en ingredientes activos superó $28^{\prime} 000.000 \mathrm{~kg} \mathrm{y}$, sólo en insecticidas fue de 3'084.750 kg; los OF ocuparon el primer lugar en producción y en importación (13).

Ante los problemas señalados,es decir, los efectos tóxicos generados por los plaguicidas, el incremento en su producción y su consumo, y el subregistro de intoxicaciones, el Ministerio de Salud estableció en 1991 la notificación obligatoria de todo caso de intoxicación o accidente ocasionado por estos productos (14). En respuesta a estas necesidades, el programa VEO ha venido desarrollándose desde hace varios años, suministrando información sobre la prevalencia de la intoxicación causada por plaguicidas OF y $\mathrm{C}$ y sobre los niveles de actividad de ACHE en la población ocupacionalmente expuesta.

\section{Materiales y métodos}

Se presenta un estudio descriptivo de la información recolectada a través del programa VEO durante 1996 y 1997, con la participación de 17 ETS del país.

Se analizaron 24.167 datos de ACHE, pertenecientes a 21.454 trabajadores ocupacionalmente expuestos a plaguicidas OF y C. Se determinaron las actividades económicas y los oficios que presentaron mayor riesgo de intoxicación. Los departamentos participantes fueron: Atlántico, Bolívar, Boyacá, Caldas, Cesar, Córdoba, Huila, Magdalena, Meta, Nariño, Norte de Santander, Quindío, Santander, Sucre, Tolima, Valle y la Secretaría de Salud de Bogotá.

Los niveles de ACHE se determinaron según el método de Limperos y Ranta modificado (7), utilizando el equipo Lovibond, mediante la obtención de una gota de sangre total por punción en el dedo medio. Este método da resultados en términos de porcentaje de la actividad de ACHE; la magnitud de la reacción se mide por la intensidad del cambio de color en la mezcla de reacción. Se consideran como casos de intoxicación los que presentan una actividad de ACHE menor de $75 \%$. Las seccionales departamentales participantes en el presente estudio hacen parte del programa interlaboratorios de control de calidad para plaguicidas OF y C (PICCCVEO), que lidera el INS con el fin de obtener una mayor confiabilidad en sus resultados.

Para definir la población que se incluye en el programa de vigilancia, anualmente se realiza un censo de las empresas cuyos trabajadores están expuestos a plaguicidas, el cual es efectuado por los promotores y técnicos de saneamiento de las respectivas secretarías de salud. Con el propósito de producir cambios de conducta en la población expuesta, el promotor realiza capacitaciones sobre el empleo seguro y eficaz de los plaguicidas, el manejo adecuado de los desechos de agroquímicos y el uso de primeros auxilios en caso de accidentes con estos productos, proporcionándoles material didáctico como afiches, plegables y cartillas.

A cada trabajador se le realiza una encuesta y se plantea la toma trimestral de una muestra de sangre. Si el valor de la ACHE es menor de $75 \%$, se adoptan medidas correctivas para obtener los cambios adecuados en los hábitos laborales. Posteriormente, el trabajador se remite al médico para su valoración y se van controlando los valores de $\mathrm{ACHE}$ hasta que se normalicen. Los resultados se consignan en un formato diseñado por el Laboratorio de Salud Ambiental del INS, el cual contiene las siguientes variables: identificación general, edad, sexo, departamento, regional, municipio, actividad económica, oficio, seguridad social y resultados de la ACHE.

En este estudio, se utilizó el programa Epi-Info para el análisis de la información y se relacionaron las variables para obtener las distribuciones de las frecuencias, ajustando las proporciones mediante el método de estandarización directa.

\section{Resultados}

Durante 1996 y 1997, se realizaron 24.167 análisis de ACHE pertenecientes a 21.454 personas con riesgo de exposición a plaguicidas OF y $\mathrm{C}$. En 
1996, participaron 13.424 personas, a las que se les realizaron 15.581 análisis y, en 1997, participaron 8.030 personas con 8.586 determinaciones de ACHE. Del total de individuos participantes en el estudio, a 19.251 (89,73\%) se les hizo una determinación de la enzima; a 1.715 (8\%) se les hicieron dos determinaciones; a 466 personas $(2,17 \%)$, tres determinaciones y a 22 personas $(0,1 \%)$, cuatro determinaciones. En relación con la población total, se efectuaron 1,1 pruebas de colinesterasa al año por persona.

Con el propósito de comparar las prevalencias de anormalidades de ACHE obtenidas en cada uno de los años de 1993 a 1997, se realizó un ajuste o estandarización directa de tasas, tomando como variable los oficios. Los cálculos se realizaron teniendo como población estándar el total de los análisis efectuados entre 1993 y 1997.Con base en estos análisis, la distribución teórica por oficio se recalculó, de manera que correspondiera a una distribución común y estándar para las poblaciones estudiadas y, así, permitir la comparación de sus prevalencias (cuadro 1). Con base en estos datos, el promedio de prevalencia para los años 1993 a 1995 fue de 6\%; para los años 1996 y 1997, de $6,2 \%$, y el del compendio de los años 1993 a 1997 fue de $6,1 \%$.

En 1996, participaron 14 ETS y en 1997, 13 ETS. Las coberturas del programa VEO, según el número de municipios en cada ETS, se presentan en el cuadro 2, así como las prevalencias de anormalidades de ACHE presentes en cada una de ellas. Con el fin de comparar las prevalencias entre las ETS, se realizó otra estandarización directa de tasas, tomando como variable los oficios. Después de realizar estos cálculos, se encontró que, en 1996, las mayores prevalencias se presentaron en Bolívar con $20,3 \%$, Córdoba con $17,1 \%$ y Santander con $16,1 \%$. En 1997, las mayores prevalencias se localizaron en Meta con $9,6 \%$, Caldas con $7,2 \%$ y Córdoba y Huila con $6,6 \%$.

Cuadro 1. Prevalencia de anormalidades de ACHE obtenidas después de realizar el ajuste de proporciones, 1993-1997.

\begin{tabular}{|c|c|c|c|c|c|c|c|c|c|c|c|c|}
\hline \multirow[b]{2}{*}{ Oficios } & \multirow[b]{2}{*}{$\begin{array}{l}\text { Población } \\
\text { estándar }\end{array}$} & \multicolumn{2}{|c|}{1993} & \multicolumn{2}{|c|}{1994} & \multicolumn{2}{|c|}{1995} & \multicolumn{2}{|c|}{1996} & \multicolumn{2}{|c|}{1997} & \multirow{2}{*}{$\begin{array}{l}93-97 \\
\bar{x} \%\end{array}$} \\
\hline & & Tasa* & * \% & Tasa* & $\%$ & Tasa* & $\%$ & Tasa* $^{\star}$ & $\%$ & Tasa $^{\star}$ & $\%$ & \\
\hline $\begin{array}{l}\text { Cargo } \\
\text { administrativo }\end{array}$ & 13.009 & 715,5 & 5,5 & 637,4 & 4,9 & 533,4 & 4,1 & 741,5 & 5,7 & 468,3 & 3,6 & 4,8 \\
\hline Secretaria & 952 & 72,4 & 7,6 & 85,7 & 9,0 & 68,5 & 7,2 & 38,1 & 4,0 & 8,6 & 0,9 & 5,7 \\
\hline $\begin{array}{c}\text { Almacenista/ } \\
\text { bodeguero }\end{array}$ & 1.411 & 18,3 & 1,3 & 119,9 & 8,5 & 69,1 & 4,9 & 101,6 & 7,2 & 122,7 & 8,7 & 6,1 \\
\hline Conductor & 628 & 5,7 & 0,9 & 23,9 & 3,8 & 25,7 & 4,1 & 37,1 & 5,9 & 22,0 & 3,5 & 3,6 \\
\hline Vendedor & 1.162 & - & - & 115,0 & 9,9 & 31,4 & 2,7 & 34,9 & 3,0 & 20,9 & 1,8 & 3,5 \\
\hline Agrónomo & 1.824 & 105,8 & 5,8 & 52,9 & 2,9 & 78,4 & 4,3 & 62,0 & 3,4 & 96,7 & 5,3 & 4,3 \\
\hline $\begin{array}{l}\text { Profesional, } \\
\text { otras ramas }\end{array}$ & 241 & 17,1 & 7,1 & 27,7 & 11,5 & 33,5 & 13,9 & 7,0 & 2,9 & 12,8 & 5,3 & 4,3 \\
\hline $\begin{array}{l}\text { Fumigador/ } \\
\text { aplicador }\end{array}$ & 19.066 & $2.040,1$ & 10,7 & $1.563,4$ & 8,2 & $1.391,8$ & 7,3 & $1.468,1$ & 7,7 & $1.258,3$ & 6,6 & 8,1 \\
\hline Jornalero & 17.432 & 453,2 & 2,6 & $1.202,8$ & 6,9 & $1.272,5$ & 7,3 & $1.516,6$ & 8,7 & 906,5 & 5,2 & 6,1 \\
\hline Piloto aviador & 78 & 3,9 & 5,0 & 16,5 & 21,1 & - & - & 12,3 & 15,8 & - & - & 5,2 \\
\hline $\begin{array}{l}\text { Mezclador/ } \\
\text { tanqueador }\end{array}$ & 412 & - & - & 71,3 & 17,3 & 105,5 & 25,6 & 70,5 & 17,1 & 66,3 & 16,1 & 15,2 \\
\hline Banderero & 48 & - & - & - & - & - & - & 16,0 & 33,3 & 9,3 & 19,3 & 10,5 \\
\hline Educador & 197 & - & - & 3,5 & 1,8 & - & - & 41,8 & 21,2 & - & - & 4,6 \\
\hline Estudiante & 7.593 & 205,0 & 2,7 & 341,7 & 4,5 & 311,3 & 4,1 & 698,5 & 9,2 & 364,5 & 4,8 & 5,1 \\
\hline Ama de casa & 4.558 & 132,2 & 2,9 & 218,8 & 4,8 & 136,7 & 3,0 & 205,1 & 4,5 & 123,1 & 2,7 & 3,6 \\
\hline $\begin{array}{l}\text { Servicios gene-. } \\
\text { rales/campo }\end{array}$ & 1.222 & 9,8 & 0,8 & 64,8 & 5,3 & 112,4 & 9,2 & 77,0 & 6,3 & 122,2 & 10,0 & 6,3 \\
\hline Otros & 524 & 10,5 & 2,0 & - & - & 52,7 & 4,9 & 61,8 & 11,8 & 65,5 & 12,5 & 8,0 \\
\hline Total & 70.357 & $3.807,8$ & 5,4 & $4.545,0$ & 6,5 & $4.195,9$ & 6,0 & $5.189,9$ & 7,4 & $3.667,7$ & 5,2 & \\
\hline
\end{tabular}

* Tasa estandarizada de anormalidades 
Cuadro 2. Entidades territoriales de salud con sus coberturas municipales y sus prevalencias de anormalidades de ACHE, 1996-1997.

\begin{tabular}{lcccc}
\hline $\begin{array}{l}\text { Entidades } \\
\text { territoriales } \\
\text { de salud }\end{array}$ & $\begin{array}{c}1996 \\
\text { Coberturas municipales }\end{array}$ & $\begin{array}{c}\text { Prevalencias } \\
\%\end{array}$ & $\begin{array}{c}\text { Coberturas municipales } \\
\%\end{array}$ & $\begin{array}{c}\text { Prevalencias } \\
\%\end{array}$ \\
\hline Atlántico & $\%$ & 2,4 & 9,1 & 3,6 \\
Bogotá & 18,2 & 9,0 & 14,3 & 0,0 \\
Bolívar & 14,3 & 45,7 & 4,8 & 7,0 \\
Boyacá & 16,7 & 4,6 & 1,6 & 5,2 \\
Caldas & 2,4 & - & 24,0 & 8,0 \\
Cesar & - & 2,6 & 1,3 & 13,5 \\
Córdoba & 8,3 & 17,8 & 16,2 & 10,3 \\
Huila & 42,3 & 5,5 & 13,6 & 9,9 \\
Magdalena & 16,2 & - & 55,2 & 7,7 \\
Meta & - & 10,8 & - & - \\
Nariño & 44,8 & 5,7 & 7,5 & 0,3 \\
Norte de Santander & 1,6 & - & - & - \\
Quindío & - & 0,0 & 2,3 & 26,3 \\
Santander & 8,3 & 17,4 & - & - \\
Sucre & 5,7 & 0,7 & - & - \\
Tolima & 45,8 & 11,9 & 92,9 & 5,2 \\
Valle & 25,5 & 4,3 & & \\
\hline
\end{tabular}

En 1996, 8.498 de los análisis (54\%) fueron informados por el Valle del Cauca. En la ETS de Bolívar, se encontró un elevado porcentaje $(45,7 \%)$ (113/247) de valores anormales de ACHE (cuadro 2 ), de los cuales $18,6 \%$ tuvo una actividad de la enzima de $37,5 \%$ o menos. También es importante anotar que en la ETS de Meta, $28,6 \%$ de los datos anormales tuvo actividades de ACHE de $37,5 \%$ o menos.

En 1997, Valle del Cauca fue la ETS que informó el mayor número de resultados (4.435 pruebas) $(51,6 \%)$. El mayor porcentaje de valores anormales de ACHE se encontró en la ETS de Santander con 26,3\% (15/57) (cuadro 2). Tanto en la ETS de Bolívar como en la de Meta, el porcentaje de afectados bajó con respecto a los informes de 1996 (cuadro 2).

Las actividades económicas con mayor porcentaje de valores anormales de ACHE entre 1993 y 1997 están consignadas en el cuadro 3. La aplicación aérea fue la actividad con mayor prevalencia de anormalidades de ACHE; además, en 1996, 48,3\% de sus pruebas tuvo actividades enzimáticas de $37,5 \%$ o menos. La aplicación aérea y la aplicación terrestre fueron dos de las actividades económicas con mayores prevalencias de valores anormales de ACHE entre 1993 y 1997.
De los 17 oficios registrados, el de banderero, aunque sólo tuvo 34 datos informados durante 1996 y 1997, presentó el mayor porcentaje de valores anormales de ACHE (cuadros 3 y 4). Fue seguido por el oficio de mezclador-tanqueadorformulador con un porcentaje similar al informado entre 1993 y 1995, años en los que este oficio presentó el mayor porcentaje de datos anormales de ACHE (cuadros 3 y 4 ).

En relación con los grupos de edad, se observó que el mayor número de datos correspondió al grupo de trabajadores de 26 a 40 años (9.407/ 24.167). Sin embargo, al igual que en la evaluación del programa VEO durante 1993-1995, el grupo de 18 a 25 años fue el que presentó la mayor prevalencia de valores anormales con 8,2\% (434/ 5.258). El segundo lugar lo ocupó el grupo de edad comprendido entre los 6 y 11 años con 7,4\% (106/ 1.427) de valores anormales de ACHE.

El 76,4\% (18.468) de los datos estudiados durante 1996 y 1997 correspondió al género masculino, con $7,2 \%$ valores anormales de ACHE. El género femenino, con 5.699 datos, presentó $6,2 \%$ (352) de valores anormales de ACHE. Esta proporción $(3,2: 1)$ tuvo una variación moderada con respecto a la proporción encontrada durante 1993-95 (4:1).

En lo referente a la seguridad social, se registraron los datos correspondientes a 24.138 personas con 
Cuadro 3. Variables que se asocian con las mayores prevalencias de ACHE anormal, 1993-1997.

\begin{tabular}{|c|c|c|c|c|c|}
\hline \multirow[t]{2}{*}{ Variables } & \multicolumn{2}{|c|}{ 1993-1995 } & \multicolumn{2}{|c|}{ 1996-1997 } & \multirow{2}{*}{$\begin{array}{c}1993-1997 \\
\%\end{array}$} \\
\hline & $\%$ & n & $\%$ & n & \\
\hline \multicolumn{6}{|l|}{ Actividades económicas } \\
\hline Aplicación aérea & 11,2 & $(82 / 729)$ & 14,0 & $(37 / 264)$ & 12,0 \\
\hline Aplicación terrestre & 15,0 & $(128 / 851)$ & 11,4 & $(27 / 236)$ & 14,3 \\
\hline Otra actividad & 11,9 & $(147 / 1238)$ & 11,4 & $(228 / 1.990)$ & 11,6 \\
\hline Formulador & 35,4 & $(17 / 48)$ & 2,6 & $(2 / 78)$ & 15,0 \\
\hline \multicolumn{6}{|l|}{ Oficios } \\
\hline Banderero & 0,0 & $(0 / 14)$ & 20,6 & $(7 / 34)$ & 14,6 \\
\hline Mezclador & 18,1 & $(49 / 270)$ & 16,9 & $(24 / 142)$ & 17,7 \\
\hline Piloto aviador & 9,6 & $(5 / 52)$ & 11,5 & $(3 / 26)$ & 10,2 \\
\hline Fumigador & 8,0 & $(680 / 8.532)$ & 7,7 & $(808 / 10.533)$ & 7,8 \\
\hline Profesionales otras ramas & 11,8 & $(18 / 152)$ & 3,4 & $(3 / 89)$ & 8,7 \\
\hline \multicolumn{6}{|l|}{ Edad } \\
\hline $18-25$ años & & & 8,2 & $(434 / 5.258)$ & \\
\hline $6-11$ años & & & 7,4 & $(106 / 1.427)$ & \\
\hline \multicolumn{6}{|l|}{ Género } \\
\hline Masculino & & & 7,2 & $(13.336 / 18.452)$ & \\
\hline \multicolumn{6}{|l|}{ Sistema seguridad social } \\
\hline Sin seguridad social & 68,3 & $(16.338 / 23.917)$ & 63,9 & $(15.414 / 24.138)$ & 66,0 \\
\hline
\end{tabular}

Cuadro 4. Total de pruebas de ACHE por oficio, 1996-1997.

\begin{tabular}{|c|c|c|c|c|c|c|c|c|c|c|c|}
\hline \multirow{3}{*}{ Oficios } & \multicolumn{4}{|c|}{1996} & \multicolumn{4}{|c|}{1997} & \multicolumn{3}{|c|}{$1996-1997$} \\
\hline & \multicolumn{2}{|c|}{ Normal } & \multicolumn{2}{|c|}{ Anormal } & \multicolumn{2}{|c|}{ Normal } & \multicolumn{2}{|c|}{ Anormal } & \multirow{2}{*}{$\begin{array}{c}\text { Total } \\
\mathbf{n}\end{array}$} & \multicolumn{2}{|c|}{ Anormal } \\
\hline & $\mathrm{n}$ & $\%$ & n & $\%$ & $\mathrm{n}$ & $\%$ & n & $\%$ & & $\mathrm{n}$ & $\%$ \\
\hline Cargo administrativo & 1.116 & 94,3 & 68 & 5,7 & 458 & 96,4 & 17 & 3,6 & 1.659 & 85 & 5,1 \\
\hline Secretaria & 284 & 95,9 & 12 & 4,1 & 106 & 99,1 & 1 & 0,9 & 403 & 13 & 3,2 \\
\hline Almacenista-bodeguero & 426 & 92,8 & 33 & 7,2 & 158 & 91,3 & 15 & 8,7 & 632 & 48 & 7,6 \\
\hline Conductor & 143 & 94,1 & 9 & 5,9 & 56 & 95,6 & 2 & 3,4 & 210 & 11 & 5,2 \\
\hline Vendedor & 385 & 97,0 & 12 & 3,0 & 160 & 98,2 & 3 & 1,8 & 560 & 15 & 2,7 \\
\hline Agrónomo & 226 & 96,6 & 8 & 3,4 & 72 & 94,7 & 4 & 5,3 & 310 & 12 & 3,9 \\
\hline Profesional, otras ramas & 68 & 97,1 & 2 & 2,9 & 18 & 94,7 & 1 & 5,3 & 89 & 3 & 3,4 \\
\hline Fumigador-aplicador & 6.162 & 91,7 & 557 & 8,3 & 3.563 & 93,4 & 252 & 6,6 & 10.534 & 809 & 7,7 \\
\hline Jornalero & 2.566 & 91,3 & 245 & 8,7 & 1.753 & 94,8 & 96 & 5,2 & 4.660 & 341 & 7,3 \\
\hline Piloto aviador & 16 & 84,2 & 3 & 15,8 & 7 & 100,0 & 0 & 0,0 & 26 & 3 & 11,5 \\
\hline $\begin{array}{l}\text { Mezclador-tanqueador- } \\
\text { formulador }\end{array}$ & 92 & 82,9 & 19 & 17,1 & 26 & 83,9 & 5 & 16,1 & 142 & 24 & 16,9 \\
\hline Banderero & 2 & 66,7 & 1 & 33,3 & 25 & 80,6 & 6 & 19,4 & 34 & 7 & 20,6 \\
\hline Profesor & 26 & 78,8 & 7 & 21,2 & 12 & 100,0 & 0 & 0,0 & 45 & 7 & 15,5 \\
\hline Estudiante & 1.604 & 90,8 & 162 & 9,2 & 963 & 95,2 & 49 & 4,8 & 2.778 & 211 & 7,6 \\
\hline Ama de casa & 1.003 & 95,5 & 47 & 4,5 & 613 & 97,3 & 17 & 2,7 & 1.680 & 64 & 3,8 \\
\hline $\begin{array}{l}\text { Servicios generales/ } \\
\text { campo }\end{array}$ & 180 & 93,8 & 12 & 6,2 & 72 & 90,0 & 8 & 10,0 & 272 & 20 & 7,3 \\
\hline Otros & 75 & 88,2 & 10 & 11,8 & 42 & 87,5 & 6 & 12,5 & 133 & 16 & 12,0 \\
\hline Total & 14.374 & 92,3 & 1.207 & 7,7 & 8.104 & 94,4 & 482 & 5,6 & 24.167 & 1.689 & 7,0 \\
\hline
\end{tabular}

riesgo de exposición a plaguicidas OF y C; de ellas, $24,3 \%$ (5.864) era cotizante del ISS, $11,8 \%$ (2.860) pertenecía a otra Entidad Promotora de Salud (EPS), y $63,9 \%$ no estaban afiliados al sistema. Este último porcentaje es levemente menor al encontrado en el programa VEO de 1993-
1995, que fue de $68,3 \%$. Es importante resaltar que $65,2 \%(1.101 / 1.689)$ de las personas con valores anormales de ACHE no pertenecía a ninguna EPS o Administradora de Régimen Subsidiado (ARS); de ellas, $66 \%$ eran hombres y $50,7 \%$, mujeres. En el cuadro 5 se presentan, 
Cuadro 5. Porcentaje de la población sin seguridad social, distribuido por edad

\begin{tabular}{|c|c|c|c|c|c|c|}
\hline \multirow[b]{2}{*}{ Edad (años) } & \multicolumn{3}{|c|}{1996} & \multicolumn{3}{|c|}{1997} \\
\hline & Total población & $\mathrm{n}$ & $\%$ & Total población & $\mathrm{n}$ & $\%$ \\
\hline $0-5$ & 135 & 89 & 65,9 & 44 & 23 & 52,3 \\
\hline $6-11$ & 843 & 685 & 81,2 & 584 & 295 & 50,5 \\
\hline $12-17$ & 1.269 & 1.108 & 87,3 & 875 & 537 & 61,4 \\
\hline $18-25$ & 3.437 & 2.428 & 70,6 & 1.817 & 1.167 & 64,2 \\
\hline $26-40$ & 6.161 & 3.640 & 59,1 & 3.226 & 1.734 & 53,7 \\
\hline $41-50$ & 2.240 & 1.445 & 64,5 & 1.221 & 672 & 55,0 \\
\hline $51-60$ & 978 & 693 & 70,9 & 547 & 326 & 59,6 \\
\hline más de 60 & 490 & 389 & 79,4 & 271 & 185 & 68,3 \\
\hline Total & 15.553 & 10.477 & 67,4 & 8.585 & 4.939 & 57,5 \\
\hline
\end{tabular}

$\mathrm{n}$ : número de personas sin seguridad social

distribuidos por edad, los porcentajes de las personas que en el momento del estudio no estaban cubiertas por el SGSSS.

\section{Discusión}

El uso creciente de los plaguicidas en una forma intensiva e indiscriminada y, en muchos casos, aplicada sin la educación ni las medidas de protección adecuadas para su manejo, ha generado importantes problemas de intoxicación en la población y de contaminación del medio ambiente en el mundo. Debido a esta situación, se ha hecho necesario el desarrollo de programas de vigilancia que permitan cuantificar y evaluar los riesgos a que están expuestas las personas que laboran directamente con estas sustancias y de las que habitan en ambientes que presentan riesgo de contaminación.

Colombia es un país agrícola que ha ido incrementando la comercialización y el uso de plaguicidas desde hace 40 años aproximadamente cuando comenzó el desarrollo de la agricultura tecnificada. Es indispensable, por tanto, conocer los niveles de intoxicación de la población expuesta y adoptar las medidas de control adecuadas a nuestra problemática. A través del programa VEO se ha avanzado en el logro de estos objetivos mediante el fortalecimiento de la vigilancia en salud pública y el desarrollo de acciones de promoción y prevención.

Las prevalencias de anormalidades de $\mathrm{ACHE}$ encontradas en el programa VEO en 1990 y en 1991 fueron 5 y $6 \%$, respectivamente (15). De 1993 a 1997, estas prevalencias oscilaron entre $5 \%$ y
$7,4 \%$ (cuadro 1), lo que probablemente explica que no hayan disminuido estos porcentajes es el fortalecimiento y el desarrollo del programa de control de calidad de los resultados analíticos de ACHE, el cual ha permitido mejorar la confiabilidad de los resultados emitidos por las ETS y definir con mayor precisión los casos de anormalidad desde que fue puesto en funcionamiento en 1993.

Otra razón es que se han retirado algunos departamentos con experiencia en la realización del programa VEO y han ingresado otros que no la tienen. Además, a medida que las ETS aumentan su cobertura, ingresan municipios en los que no se ha llevado a cabo la vigilancia de plaguicidas. Estos municipios presentan porcentajes de intoxicación más altos que los del promedio de los participantes, debido a que las labores de prevención y control se inician al poner en marcha el programa.

A pesar de estos hechos, las prevalencias no han aumentado y sus valores son muy similares al 4,8\% informado en 1989 por el Programa de Vigilancia Epidemiológica de California, al 5,4\% encontrado en el estudio de mediciones de ACHE realizado en 1993 también en California y al 8,3\% del estudio llevado a cabo en Taiwán (8).

El programa ha contado con la participación constante de la mayoría de los departamentos que tienen cultivos permanentes; por tanto, se han podido obtener datos significativos sobre la situación de la contaminación con plaguicidas inhibidores de la ACHE. Sin embargo, para establecer la verdadera magnitud de la intoxicación por OF y $\mathrm{C}$ y el impacto ambiental que producen, 
es indispensable aumentar la cobertura del programa.

En cuanto a las ETS, la cobertura fue en aumento hasta 1993, cuando llegó a incluir 20 departamentos (15), pero, a partir de 1994, ha ido disminuyendo debido al proceso de descentralización administrativa, a la puesta en marcha del SGSSS y a la poca claridad que en un comienzo presentó el Programa de Atención Básica (PAB). Sin embargo, la cobertura municipal ha ido aumentando de manera significativa en algunos departamentos, como Valle del Cauca, que ya alcanzó el 100\%, Caldas, Huila y Meta.

A través de la evolución del programa se ha observado que más de las tres cuartas partes de los datos analizados corresponde al género masculino (debido a que la mayoría de los oficios son realizados por hombres); sin embargo, el riesgo de intoxicación por OF y C es muy similar entre los dos géneros $(7,2 \%$ para el género masculino y $6,2 \%$ para el femenino).

Con respecto a la edad, la mayor prevalencia de valores anormales de $\mathrm{ACHE}(8,2 \%)$ se encontró en el grupo de 18 a 25 años, lo cual era de esperar, puesto que $75 \%$ de los datos pertenecientes a este rango de edad corresponden a trabajadores con oficios de alto riesgo de exposición. A diferencia del primero, el segundo lugar fue ocupado por un grupo de edad del que se esperaría un bajo riesgo de exposición y fue el de 6 a 11 años. La alta prevalencia de valores anormales de ACHE en este grupo de edad se debió a un agrupamiento (cluster) detectado en el departamento de Santander en 1996.

En cuanto a la participación, es importante resaltar el grado de respuesta del Valle del Cauca, que durante los años de funcionamiento del programa ha sido la ETS con mayor cobertura de municipios, de población y de exámenes realizados. Bogotá y Cesar, manteniendo los mismos niveles de cobertura durante 1996 y 1997, presentaron una notable disminución en las prevalencias de valores anormales de ACHE. Aunque por la información que tenemos no nos es posible concluir acerca de las causas que motivaron este descenso, es probable que las acciones de promoción y prevención en las respectivas ETS hayan influido en la obtención de estos logros.

La realización de cuatro determinaciones de ACHE a cada trabajador por año, que era uno de los objetivos del programa, no se cumplió debido a la crisis administrativa y económica de las ETS. La reestructuración de las ETS llevó a la disminución de los recursos destinados al programa VEO y a la reducción del personal. Algunos funcionarios con experiencia en el manejo del programa fueron despedidos y los que quedaron no alcanzaron a cumplir los objetivos a cabalidad. Mientras esta situación persista, el seguimiento de determinaciones de ACHE sólo se realizará a los trabajadores con valores anormales.

De los datos analizados, se destaca el bajo número de afiliaciones a la seguridad social. Esta misma situación se aplica a los dos géneros y a todos los grupos de edad. Por otra parte, en las ETS, los porcentajes de la población expuesta sin afiliación a la seguridad social, oscilan entre 0 y $88,6 \%$. Nueve de los 17 departamentos tienen más de $50 \%$ de la población sin afiliación a la seguridad social. Esta observación es muy importante para las autoridades que tienen a su cargo el régimen subsidiado en el SGSSS.

Los datos presentados indican que en el país se hace necesario revisar las afiliaciones al sistema de seguridad social, puesto que la mayoría de las personas expuestas a los riesgos de contaminación detectados por el programa, se encuentran desprotegidas en la atención de su salud. Esta situación puede llevar a agravar las intoxicaciones que se presenten por falta de atención oportuna o por ausencia de atención. En este último caso, surge otra consecuencia y es el incremento del subregistro, uno de los mayores impedimentos para conocer las verdaderas dimensiones de los problemas de salud en el país.

Con el objeto de fortalecer el programa y desarrollar mayores y mejores acciones de promoción y prevención, es importante que las diversas ETS mantengan en operación permanente la vigilancia epidemiológica para plaguicidas inhibidores de la ACHE y envíen trimestralmente los informes de actividades y los resultados correspondientes; que se asigne y se mantenga la continuidad de los 
funcionarios que manejan el programa, de manera que las capacitaciones y experiencias adquiridas sean bien aprovechadas; que los directores de los hospitales municipales y locales adquieran una mayor conciencia de los beneficios de este programa de vigilancia para que incrementen el apoyo a los supervisores y técnicos en el cumplimiento de sus funciones; que se incrementen las campañas de prevención, se mejore el suministro de la dotación que le corresponde a cada empleado y que ellos, por su parte, establezcan hábitos laborales adecuados; que se modifique el formato de inscripción de resultados que cada seccional remite al INS, con la inclusión de otras variables para precisar, enriquecer y actualizar la información relacionada con el funcionamiento del programa, y encontrar más asociaciones entre los grados de intoxicación y los plaguicidas utilizados; que se publiquen estudios individualizados por departamentos, en los que se analicen los resultados y la trayectoria que ha tenido cada uno de ellos a lo largo de su participación en el programa.

Con la presente publicación, el INS está dando cumplimiento a uno de los requisitos establecidos en el convenio de cooperación y asistencia tecnico-científica para el desarrollo del programa VEO y, aunque en el país todavía no se dispone de cifras exactas sobre las intoxicaciones relacionadas con el uso de OF y $\mathrm{C}$, este programa de vigilancia, que es el único que se realiza a nivel nacional, continúa aportando datos valiosos y de aplicación inmediata para el conocimiento y el control de las intoxicaciones producidas por plaguicidas inhibidores de la colinesterasa.

Invitamos a las seccionales de salud que no han participado o que lo han hecho en forma discontinua, para que se inscriban y participen en el programa de vigilancia de OF y $\mathrm{C}$, con el propósito de obtener información confiable que permita abordar la problemática en sus verdaderas dimensiones.

\section{Agradecimientos}

De manera muy especial agradecemos a los coordinadores del programa VEO en cada una de las ETS, al igual que a los supervisores y técnicos de saneamiento, quienes a pesar de las limitaciones existentes, cumplieron con la participación en el programa de vigilancia de plaguicidas durante 1996 y 1997. De igual manera, queremos agradecer el apoyo de las directivas de hospitales y secretarías de salud que han contribuido a la protección de los trabajadores expuestos a plaguicidas OF y $\mathrm{C}$ y han prevenido la ocurrencia de intoxicaciones.

\section{Referencias}

1. García JE. Intoxicaciones agudas con plaguicidas: costos humanos y económicos. Rev Panam Salud Pública 1998;4:383-7.

2. Lotti M. Cholinesterase inhibition: complexities in interpretation. Clin Chem 1995;41:1814-8.

3. Idrobo AJ. Intoxicaciones masivas con plaguicidas en Colombia. Biomédica 1999;19:67-76.

4. Michel HD. An electrometric method for the determination of red blood cell and plasma cholinesterase activity. J Lab Clin Med 1949;34:1564-8.

5. Vallejo MC. Química y análisis de plaguicidas. En: Toxicología general. Bogotá: Consejo Colombiano de Seguridad; 1989 p.288-92.

6. Limperos G, Ranta KE. A rapid screening test for the determination of the approximate cholinesterase activity of human blood. Science 1953;117:453-5.

7. Edson EF. Measurement of cholinesterase activity of whole blood. Brit Med J 1955;i:1218.

8. Fillmore $\mathbf{C M}$, Lessenger JE. A cholinesterase testing program for pesticide applicators. J Occup Med 1993; 35:61-70.

9. Henao S, Corey G. Plaguicidas y organofosforados carbámicos. Serie vigilancia 2. Metepec, México: Centro Panamericano de Ecología Humana y Salud, OPS, OMS; 1986. p.28.

10. Wesseling C, Castillo L, Elinder CG. Pesticide poisoning in Costa Rica. Scand J Work Environ Health 1993; 19:227-35.

11. Varona M, Morales L, Ortiz J, Sánchez J, Cárdenas O, de la Hoz F. Panorama epidemiológico de exposición a plaguicidas inhibidores de colinesterasa en 17 departamentos del país. Biomédica 1998;18:22-9.

12. Morales L, Silva E, Ortiz JE, Ramírez P, García A. Intoxicación por plaguicidas en el departamento del Valle del Cauca, 1997. Inf Quinc Epidemiol Nac 1998;3:222-5.

13. Instituto Colombiano Agropecuario. Comercialización de plaguicidas 1994-1995: importación-producciónventas-exportación. Bogotá: ICA; 1997. p.9-12.

14. Ministerio de Salud. Disposiciones sanitarias sobre uso y manejo de plaguicidas. Decreto 1843 (22 de julio de 1991).

15. Toro G. Hombre, hambre y contaminación del medio ambiente. Rev Fac Med U Nal Col 1993;41:28-35. 